\title{
EL MODELO SEMICUÁNTICO DE BOHR EN LOS LIBROS DE TEXTO
}

\section{The Bohr's quantum model in the textbook}

\author{
Jorge Eliécer Moreno Ramírez ${ }^{1}$ \\ Rómulo Gallego Badillo \\ Royman Pérez Miranda ${ }^{3}$
}

\begin{abstract}
Resumen: La didáctica de las ciencias naturales está cuestionando la transposición de los modelos científicos en modelos didácticos. Otras investigaciones muestran que el conocimiento científico convertido en conocimiento escolar produce deformaciones de la actividad científica y la simplificación de modelos, contribuyendo con la actitud negativa de los estudiantes hacia las ciencias y al fracaso escolar reportado por algunos estudios. Se pregunta si el modelo atómico de Bohr que se muestra en los textos se corresponde histórica y epistemológicamente con las actividades científicas y cuáles son las posibles deformaciones que de ésta se hace cuando se muestra el modelo en los libros. Los resultados de la investigación muestran una clara diferencia entre la propuesta de Bohr (1913) y la transposición del modelo, lo que invita a una reflexión profunda acerca de la confiabilidad que pueden tener los textos utilizados para la enseñanza de la ciencia química

Palabras clave: Transposición didáctica. Textos. Confiabilidad. Historia de la Ciencia.
\end{abstract}

\begin{abstract}
Natural science teaching is questioning the transposition that is made of scientific models into didactic models, because research has shown that when scientific knowledge is transformed into scholarly knowledge in order to be taught it causes the deformation of the scientific activity and the simplification of the models. This contributes to the negative attitude of students towards science, and student failure shown in some studies. This research inquired if the transposition of Bohr's atomic model that is shown in textbooks corresponds historically and epistemologically to scientific activity and what the possible deformations are that, coming from this model, are made to the scientific activity that comes from the transposition. The research results show differences between what Bohr (1913) proposed and the transposition of the model in textbooks. This information will contribute to the analysis that is being done regarding the dependability of textbooks.
\end{abstract}

Keywords: Didactics transposition. Science texts. Reliability. History of Science.

\footnotetext{
${ }^{1}$ Licenciado en Química, magister en docencia de la Química. Universidad Pedagógica Nacional. Bogotá, D.C. Colombia.<Jor_31534@yahoo.es>

${ }^{2,3}$ Licenciados en Química, magister en Educación. Docentes, Departamento de Química, Universidad

Pedagógica Nacional. Bogotá, D.C., Colombia. <rgallego@uni.pedagogica.edu.co>;

royman@uni.pedagogica.edu.co
}

${ }^{1}$ Diagonal $82 \mathrm{~B}$ n ${ }^{\circ} 76 \mathrm{~d}-10$

Bogotá, D.C., Colombia 
Moreno Ramírez, J. E.; Gallego Badillo, R.; Pérez Miranda, R.

\section{Introduccion}

Dos consideraciones iniciales: por un lado, el libro de texto es una fuente de consulta permanente cuando se enseña química por lo que es de esperarse que la forma en que éstos presenten los hechos que dieron lugar a la necesidad de elaborar, estudiar, aceptar y abandonar modelos científicos en el seno de las comunidades de especialistas tenga un efecto didáctico en la enseñabilidad (GALLEGO BADILLO; PÉREZ MIRANDA, 1998) y la imagen de ciencia que promueven (GALLEGO BADILLO et al., 2004) y por otro, la presencia de capítulos dedicados a la historia de la ciencia en general y de los modelos atómicos en los textos en particular, muestra la importancia que se le da al desarrollo histórico de la química puesto que se considera que los estudiantes que aprenden historia de la ciencia comprenden mejor la ciencia escolar (Salomón, 1992, citado por Garritz, 2006); este aspecto es objeto de critica, reflexión didáctica e investigación en el interior del grupo IREC, por cuanto se ha determinado en otras investigaciones (CAMACHO GONZÁLEZ; PÉREZ MIRANDA, 2005; CUÉLLAR FERNÁNDEZ; GALLEDO BADILLO; PÉREZ MIRANDA, 2008; CAMACHO GONZÁLEZ; GALLEGO BADILLO; PÉREZ MIRANDA, 2007; CUÉLLAR FERNÁNDEZ; GALLEGO BADILLO; PÉREZ MIRANDA, 2005; GALLEGO BADILLO et al., 2004; HERREÑO CHÁVEZ; GALLEGO BADILLO; PÉREZ MIRANDA, 2010; PÁEZ; RODRÍGUEZ; NIAZ, 2004) que el saber sabio transformado en saber escolar expone un enfoque deformado de la actividad científica (FERNÁNDEZ et al., 2002) y la simplificación de modelos, como consecuencia de la narración historiográfica que se hace de los acontecimientos históricos de la ciencia química, que a juicio de los autores de los libros, son los relevantes para ser contados, como lo muestra la siguiente cita en un texto utilizado en la formación inicial de profesores: “[...] el tratamiento de Bohr es muy complejo y no se considera correcto en todos sus detalles. Por ello aquí sólo se concentrará en los planteamientos importantes y en los resultados finales que explican la posición de las líneas espectrales" (CHANG, 2007, p. 274).

Lo que le brinda al lector una imagen de ciencia acumulativa, deja de lado las controversias, las dificultades y las limitaciones que son inherentes a la formulación de los modelos científicos; se muestra una posición propia del empìro - inductivismo dentro de una lógica positivista, posiciones que son cuestionadas desde los estudios epistemológicos actuales. La investigación buscó establecer si la transposición didáctica (CHEVALLARD, 1991) que se hace del modelo semicuántico de Bohr en algunos textos de enseñanza utilizados en la formación inicial de profesores de química y en educación media, originan una visión deformada de la actividad científica y la simplificación de la propuesta inicial de Bohr, los resultados aportarán a la reflexión que se viene haciendo respecto de la confiabilidad de los libros de texto en cuanto a la versión de ciencia que promueven.

Para solucionar el problema generado por la transposición didáctica, se hace necesario que se incorpore el estudio histórico (SOLBES; TRAVER, 1996) y epistemológico en la enseñanza de las ciencias con el fin de superar la superficialidad con la que se vienen tratando los aspectos históricos que aparecen en los libros utilizados para la enseñanza. Se hace necesario mostrar los procesos que hicieron emerger, modificar y abandonar modelos en las comunidades de especialistas; las implicaciones y confrontaciones propias de toda actividad científica con el fin de romper con la imagen de ciencia ahistórica, aproblemática, lineal, acumulativa y 
El modelo semicuántico de Bohr ...

teleológica; versión ya superada desde los estudios de la epistemología que explicó con Kuhn (1972) y Lakatos (1998) los fundamentos de la física como ciencia y que con Tomasi (1999), Caldin (2002), Scerri (2003) y Del Re (2000) discuten el estatuto científico de la ciencia química desde la categoría epistemológica de modelo. Caldin (2002) clasifica los modelos como icónicos o pictóricos cuando se refieren a fenómenos no observados directamente por los investigadores; analógicos cuando son una representación idealizada de un sistema a estudiar y simbólicos cuando son producto de la axiomatización matemática de un objeto de conocimiento. Desde la investigación se admitió que la ciencia química es producto de la elaboración, aceptación, modificación, abandono y sustitución de modelos por otros con mayor potencial explicativo (GALLEGO BADILLO et al., 2006) y el modelo semicuántico de Bohr se correspondió con un modelo analógico - simbólico.

\section{De las deformaciones de la ciencia}

Para la enseñanza del saber científico generalmente se acude a los libros como mediadores entre el conocimiento científico y lo que se debe enseñar puesto que gozan de una alta confianza y aceptabilidad por parte de los profesores y estudiantes pues se considera que allí están los contenidos que con mayor pertinencia facilitarían el aprendizaje efectivo de las ciencias. Investigaciones realizadas (CUÉLLAR FERNÁNDEZ; GALLEGO BADILLO; PÉREZ MIRANDA, 2008; GÁNDARA GOMEZ; GIL QUÍLEZ; SANMARTÍ PUIG, 2002; GONZÁLEZ RODRÍGUEZ; GARCÍA BARROS; MARTÍNEZ LOSADA, 2003), muestran que la transposición, en general, de la ciencia y en particular de los modelos, llevan consigo tergiversaciones que por un lado dificultan el aprendizaje y por otro muestran una forma de hacer ciencia que no corresponde a la labor científica generando obstáculos epistemológicos en el proceso de enseñanza y aprendizaje de la ciencia.

Fernández et al. (2002) establecen varias deformaciones de la actividad científica, a saber: a) una concepción aproblemática y ahistórica de la ciencia, ya que no muestran los problemas a los que se enfrentan las comunidades científicas al momento de investigar; los modelos se presentan ya elaborados sin mostrar las limitaciones propias de la investigación; aparentemente las leyes, teorías o modelos no surgen de problemas por solucionar sino de observaciones juiciosas de los científicos sin ninguna conexión con el desarrollo histórico en el que se desenvuelven las comunidades científicas; b) una concepción empiro - inductivista y ateórica de ciencia, en la que se le da relevancia a la observación y a la experimentación como artífices de la investigación científica dejando de lado el papel de las hipótesis y de los cuerpos teóricos como soportes de la investigación; c) una concepción rígida de la actividad científica en la que personajes brillantes y aislados del mundo producen el conocimiento observando y experimentando con un método infalible, indagando por las leyes ocultas en la naturaleza, las cuales son presentadas posteriormente como algoritmos exactos e infalibles producto del control cualitativo riguroso que se hizo del proceso haciendo que la creatividad y la inventiva no tengan cabida en la actividad científica; d) una concepción exclusivamente analítica de la ciencia, la cual ignora los intentos de unificación y de construcción de cuerpos coherentes de conocimiento debido a la parcelación y acotación de los estudios; e) una concepción acumulativa del desarrollo científico en donde el conocimiento se apila a la manera de una pirámide y 
Moreno Ramírez, J. E.; Gallego Badillo, R.; Pérez Miranda, R.

las teorías o leyes recientemente descubiertas tiene un mayor poder de explicación sobre los demás conceptos, ignorando las crisis y sus consecuencias como la remodelación de los modelos aceptados inicialmente; f) una concepción individualista y elitista de la ciencia en donde los científicos son genios aislados que aparecen de vez en cuando para hallar las explicaciones que se encuentran en la naturaleza lo que deja de lado el trabajo y el intercambio de información por las comunidades científicas y g) una visión descontextualizada, socialmente neutra de la actividad científica, los científicos son ajenos a las influencias que puedan ejercer del mundo exterior y por lo tanto sus resultados no tendrían ninguna implicación social, cultural, económica ni ambiental.

\section{El modelo semicuántico de bohr, una recostrucción histórica}

La inquietud por el comportamiento de los electrones ya se había hecho evidente en los trabajos de Bohr, en mayo de 1911 defiende su tesis doctoral relacionada con la conductividad y otras propiedades metálicas de las partículas libres, enuncia en un primer momento la dificultad de la mecánica clásica para explicar el comportamiento de estas partículas. En la misma época se estaba trabajando sobre el aislamiento del electrón, Lorentz había publicado el primer desarrollo sistemático de la mecánica estadística de los electrones libres fundamentado en dos principios: a) en ausencia de campos externos o diferencias de temperatura los electrones y los átomos permanecen en equilibrio mecánico, es decir, sólo en presencia de una fuerza electromotriz los electrones se desplazarían generando una corriente eléctrica y b) si no actúan fuerzas externas, los átomos actúan isotrópicamente sobre los electrones libres (LORENTZ, 1905). Supone que los electrones rebotan elásticamente cuando chocan con los átomos metálicos a la manera de bolas de billar y que los efectos de las colisiones son insignificantes, aunque sus efectos se manifiestan en forma de calor. Bohr se enfrenta a un gran desafío: tratar de realizar los cálculos de los electrones libres de los metales consistentemente con la teoría de Lorentz. (HEILBRON; KUHN, 1969).

Luego de terminar sus estudios en Copenhague viaja a Inglaterra y decide ir a Cambridge, pues considera que allí está el centro de la física y a que siente admiración por J. J. Thomson, director del Cavendish Laboratory a quien considera un hombre maravilloso. A su arribo al centro de investigación intercambia algunas ideas sobre radiación y magnetismo con Thomson. A pesar de las expectativas, Bohr no toma la plaza ofrecida, probablemente su decisión se debió que Thomson no le prestó la suficiente atención (HEILBRON; KUHN, 1969), sin embargo su paso por Cambridge le permitió tener acceso a información de su interés investigativo.

J. J. Thomson y su grupo de colaboradores habían elaborado un modelo atómico producto del estudio de los rayos catódicos (THOMSON, 1897). La propuesta consistía en una esfera con electrificación positiva uniforme que es neutralizada por una masa de electrones negativos que se encuentran en anillos coplanares y concéntricos a la esfera y con densidad de carga constante (THOMSON, 1904). A pesar de la artificialidad del modelo, Thomson lo emplea para explicar cualitativamente la tabla periódica, la combinación química y la dispersión de los rayos alfa, igualmente introduce una explicación para el efecto fotoeléctrico desde el "modelo del doblete", según Heilbron y Kuhn (1969) este aspecto interesó a Bohr, en razón 
a que en este modelo un electrón describiría una orbita circular bajo la influencia de un momento bipolar eléctrico $\boldsymbol{P}$, no obstante que no todas las orbitas podían ser calculadas, lo que llamó la atención del investigador fue que un valor incorporado por Thomson se correspondía con la constante de Planck multiplicada por tres y que luego de realizados todos los cálculos, los valores coincidían con $\boldsymbol{h} \boldsymbol{v}$, la ecuación de Planck.

Bohr arribó al grupo de Manchester en 1912 liderado por E. Rutherford, allí comenzó a trabajar con aspectos relacionados con la radiactividad, trabajo que también desarrollaban Geiger y Marsden. En el seno de este grupo se propuso un modelo atómico con núcleo positivo y un sistema de electrones girando a cierta distancia de él (RUTHERFORD, 1911), el modelo fue ideado luego del análisis del experimento de desviación de partículas $\alpha$ (alfa) que habían realizado Geiger y Marsden (1909, 1913). Para Bohr, el átomo nuclear es la mejor forma de explicar las desviaciones observadas de las partículas $\alpha$ :

[...] Great interest is to be attributed to this atom-model; for, as Rutherford has shown, the assumption of the existence of nuclei, as those in question, seems to be necessary in order to account for the results of the experiments on large angle scattering of the a rays $[\ldots]$. (BOHR, 1913, p. 1)

Con esta afirmación muestra su desacuerdo con el modelo de Thomson (1904) y se inclina por la propuesta de Rutherford por estar mejor corroborada por la experiencia, no obstante, cuestiona la inestabilidad del sistema de electrones del modelo nuclear, siendo este aspecto el punto de partida para la elaboración del modelo semicuántico.

In an attempt to explain some of the properties of matter on the basis of this atom-model we meet however, with difficulties of a serious nature arising from the apparent instability of the system of electrons: difficulties purposely avoided in atom-models previously considered, for instance, in the one proposed by Sir J. J. Thomson.* According to the theory of the latter the atom consists of a sphere of uniform positive electrification, inside which the electrons move in circular orbits. (BOHR, 1913, p. 1)

Para Bohr, un cálculo sencillo mostraría que el electrón girando alrededor del núcleo irradiaría energía continua haciendo que las orbitas que describirían las partículas comenzaran a contraerse hasta tomar posiciones cercanas al núcleo como consecuencia del aumento de la frecuencia y a la ganancia de energía cinética. Los átomos no funcionan así en la naturaleza, por el contrario, presentan dimensiones y frecuencias fijas; concluye que la electrodinámica clásica no es adecuada para explicar el comportamiento de sistemas atómicos e introduce un valor extraño a la electrodinámica clásica para explicar la estabilidad del sistema de electrones: la constante de Planck o de los cuantos elementales, considera que el tamaño de la constante en cuestión, permite una mejor relación con la masa y carga de las partículas observadas.

El modelo semicuántico tiene un núcleo positivo con un electrón de masa muy pequeña comparada con la del núcleo y se mueve a velocidades menores a la de la luz, con orbitas 
Moreno Ramírez, J. E.; Gallego Badillo, R.; Pérez Miranda, R.

estacionarias circulares y cerradas a cierta distancia del núcleo, de no existir una perturbación externa un electrón en estado estacionario no emite energía y posee un valor que corresponde a un número entero $t$, si $\mathrm{t}=1$ la energía cinética del electrón es mayor por lo tanto el valor igual a 1 es el estado mas estable del sistema núcleo - electrón. Introduciendo la constante de Planck en los cálculos. Bohr nota que los resultados concuerdan con otros valores como los del potencial de ionización, frecuencia óptica y las dimensiones lineales de los átomos. Destáquese que el trabajo desarrollado por J. W. Nicholson en 1912 ya proponía un átomo con anillos donde se mueven los electrones y hacia un acercamiento para explicar las líneas observadas en nebulosas lejanas y en la superficie solar, relacionándolas con la teoría de Planck, lo que marcó el trabajo que Bohr desarrollaría posteriormente (HEILBRON; KUHN, 1969).

El modelo es sustentado con base en planteamientos matemáticos que le permitieron explicar las líneas del hidrógeno observadas por Balmer (1885) como radiación homogénea emitida durante la transición entre estados estacionarios con un valor igual a la relación entre ésta y la frecuencia $\mathrm{W}_{t 2}-\mathrm{W}_{t 1}=\mathrm{hv}$ en donde $\mathrm{h}$ es la constante de Planck y $t$ es un número entero. Un sistema atómico nuclear y un electrón están en equilibrio cuando el momento angular de los electrones es igual a un número entero múltiplo de h/2ð. Para hacer corresponder el modelo con la ley de Kirchhoff de la radiación térmica, los espectros de absorción tienen la misma frecuencia de los espectros de emisión; la condición para que un único electrón absorba energía es que debe estar en un estado estacionario A2 cuyo valor es menor comparado con un estado estacionario A1, porque en condición ordinaria el hidrógeno no absorbe radiación, haciéndolo únicamente cuando el gas está en estado luminoso

Las dificultades matemáticas del modelo relacionadas con las trayectorias elípticas fueron solucionadas por Sommerfeld (LAKATOS, 1998), sin embargo, los cálculos no permitían establecer un aumento en el número de orbitas obtenidas por el método de Bohr lo que impedía incrementar el número de niveles de energía posibles para el modelo, Sommerfeld corrige los cálculos con base en la teoría de la relatividad de Einstein, en efecto, los cálculos relativistas dieron los resultados esperados explicando la estructura fina del espectro del átomo de hidrogeno (DE BROGLIE, 1952). Sin embargo algunas líneas previstas por Sommerfeld para los espectros ópticos y los rayos X no fueron observadas por lo que se elaboró el principio de exclusión (PAULI, 1925) para su explicación, igualmente el modelo no explicaba el efecto Zeeman (1897) según el cual, el espectro luminoso se afectaba por la influencia de un campo magnético para lo que Goudsmit y Uhlenbeck (1926) desarrollan la hipótesis del spin, en ella los electrones girarían sobre su propio eje, con un momento magnético y momento angular $\mathbf{r}$ propios (LAKATOS, 1988)

\section{De las controversias}

Fowler reprodujo en laboratorio, con hidrógeno y helio, un espectro observado en el Sol en 1898, ya reportado por E. Pickering en 1896 en la estrella æ Puppis (LAKATOS, 1998), las líneas, que en un comienzo se aceptaron como series del Hidrógeno, no coincidían con el modelo propuesto. Para resolver la cuestión, Bohr elabora un modelo de helio ionizado el cual, produciría la serie Fowler, el papel dado al hidrógeno fue el de aceptor de un electrón, ya que éste podía tomar carga negativa. Propone que el mismo experimento pero utilizando cloro 
El modelo semicuántico de Bohr ...

como catalizador daría los mismos resultados, el modelo fue corroborado. Fowler aceptó que el espectro por él observado correspondía al helio ionizado sin embargo puntualizó que el ajuste hecho por Bohr igualmente fracasaba, ya que las longitudes de onda de la serie Fowler difieren de las calculadas para el Helio. Bohr respondió con un ajuste de las masas reducidas, el modelo sale airoso nuevamente (LAKATOS, 1998). Otro aspecto que jugó a favor del modelo semicuántico de $\mathrm{N}$. Bohr es la corroboración matemática que se puede hacer de la fórmula empírica de J. Rydberg (TIPPENS, 1988).

Bohr lanza una idea atrevida para la época, postula que el modelo se debe interpretar desde dos teorías: una en la que el equilibrio dinámico de los estados estacionarios podría ser discutido con la mecánica clásica y la transición de un estado estacionario a otro debe tratarse con la teoría de M. Planck,

It will now be attempted to show that the difficulties in question disappear if we consider the problems from the point of view taken in this paper. Before proceeding it may be useful to restate briefly the ideas characterizing the calculations on p. 5. The principal assumptions used are: (1) that the dynamical equilibrium of the systems in the stationary states can be discussed by help of the ordinary mechanics, while the passing of the systems between different stationary states cannot be treated on that basis.

(2) That the latter process is followed by the emission of a homogeneous radiation, for which the relation between the frequency and the amount of energy emitted is the one given by Planck's theory. (BOHR, 1913, p. 1)

Esta afirmación le ocasiona resistencia de la comunidad científica, entre los críticos destacan investigadores como J. J. Thomson (PÁEZ; RODRÍGUEZ; NIAZ, 2004), De Broglie, Schrödinger y Heisenberg. De Broglie (1952) cuestiona que el modelo no puede precisar la totalidad de la naturaleza, las intensidades y el estado de polarización de la radiación; considera artificial la alianza entre la dinámica clásica y la teoría cuántica, por cuanto los electrones tendrían un comportamiento caótico y contradictorio; Schrödinger (2001) discute que el modelo sólo es aplicable a sistemas muy simples como el hidrógeno, critica su origen y la dualidad planteada entre mecánica clásica y mecánica cuántica, pues no se especifica el tiempo en el que se hacen las transiciones al no explicar el estado en que el sistema se halla durante la mayor parte del tiempo. Por su parte, Heisenberg (1927) cuestiona la credibilidad que puede tener la existencia de orbitas como lo postula Bohr, puesto que no se podía medir su existencia, lo cual es refutado por Einstein, pues plantea que es la teoría la que dice qué se debe observar (HEISENBERG, 1979).

Para defender el modelo, Bohr (1921) elabora el principio de correspondencia según el cual, la electrodinámica clásica explicaría los fenómenos desde un componente macroscópico; lo cuántico explicaría los fenómenos microscópicos, esos fenómenos macroscópicos son resultado de fenómenos cuánticos elevados, por lo que si el electrón de un átomo nuclear se encuentra girando a una distancia considerable del núcleo emite energía en una serie continua de frecuencias; en el átomo cuántico, las transiciones que pueden afectar el electrón que se 
Moreno Ramírez, J. E.; Gallego Badillo, R.; Pérez Miranda, R.

encuentra en orbitas estacionarias, estaría en correspondencia con cada una de las frecuencias del átomo de Rutherford.

El modelo de Bohr resolvió la inestabilidad del sistema de electrones del modelo de Rutherford, explicó las líneas de Balmer (1885) y Paschen (1908) y predijo las líneas de Lyman (1914), Brakett (1922) y Pfund (1924); sin embargo enfrentó dificultades relacionadas con los espectros de los rayos $\mathrm{X}$, con las moléculas diatómicas y los pares inexplicados del álcali (LAKATOS, 1998); la aplicación a átomos de un sólo electrón, la precisión para explicar las intensidades y polarizaciones de las radiaciones monocromáticas y la inclusión de la mecánica y la electrodinámica clásica con los cuantos de Planck llevaron a que el modelo semicuántico de N. Bohr fuera abandonado a pesar de los esfuerzos de un connotado grupo de investigadores y que se abriera paso la mirada ondulatoria de De Broglie postulada en 1925 (1952), la mecánica de matrices de Heisenberg (1927) que borró el concepto de partícula y por lo tanto el de órbita y la mecánica ondulatoria propuesta en 1925 por E. Schrödinger.

\section{Metodología}

Para desarrollar la investigación propuesta la metodología se estructuró en tres fases: a) el estudio de los documentos publicados en la Web por el profesor Carmen Giunta del Lemoyne Collegue de New York, con el fin de caracterizar histórica y epistemológicamente el proceso que dio lugar a la emergencia del modelo semicuántico de Bohr; b) de este análisis se establecen los descriptores, subcategorías y categorías epistemológicas a utilizar para la investigación (Anexo A) y c) la selección de los libros, lo cual se hizo con base en una entrevista abierta que se realizó a profesores de educación media de varios colegios públicos de Bogotá, D. C. y a la revisión del Syllabus del programa curricular de química de La Universidad Distrital Francisco José de Caldas de Bogotá, D.C. para primer y tercer semestre de profesores en formación y que trataran el tema a investigar, se escogieron un total de 10 Libros sugeridos en la formación inicial de profesores (LFP) y 7 textos para educación media (LEM) (Anexo B).

Las claves de análisis se elaboraron de acuerdo con los siguientes criterios, RS: Referencia satisfactoria, cuando la referencia hecha en los libros de texto se acercara a una elaboración histórico -epistemológica aceptable; R: Referencia descriptiva en la Retórica de conclusiones, (NÍAZ, 2005), cuando la referencia hecha en el texto se limitara a una descripción sin elementos de tipo histórico - epistemológico; RI: Referencia incompleta, cuando la referencia se hace descriptivamente y se omiten aspectos relacionados con el modelo a estudiar; SR: Sin referencia, cuando no aparece ninguna mención de algún aspecto histórico - epistemológico relacionado con el modelo y $\mathrm{r}$ cuando se hace una referencia errónea en el texto; se asignó un valor numérico para cada clave: RS: 5; R: 4; RI: 3 y SR y r: 0 con el fin de cuantificar cuánto se acerca o se aleja el modelo presentado en los texto del caracterizado en la investigación.

Ejemplo de una referencia satisfactoria:

En los años que van de 1911 a 1913 existió gran incertidumbre acerca de la estructura atómica. Se había descartado el modelo de Kelvin - 
El modelo semicuántico de Bohr ...

Thomson (el cual predecía estabilidad para los átomos) por otro que estaba de acuerdo con los experimentos de dispersión de partículas á, pero que, además de ser inestable, no concordaba con la espectroscopia experimental.

En 1913 Niels Bohr desarrolló un modelo atómico abandonado las consideraciones de la electrodinámica clásica y tomando en cuenta la cuantización de la energía en la interacción radiación-materia, introducida por Max Planck en 1900. (LFP 3, p. 156)

Ejemplo de una referencia descriptiva - Retórica de conclusiones (NÍAZ, 2005):

En 1913, poco después de los descubrimientos de Planck y de Einstein, el físico danés Niels Bohr dio a conocer una explicación teórica del espectro de emisión del átomo de hidrogeno. Cuando Bohr abordó por primera vez este problema, los físicos ya sabían que los átomos estaban formados por electrones que giraban alrededor del núcleo en orbitas circulares a gran velocidad. (LFP 4, p. 274-275)

Ejemplo de una referencia incompleta:

En 1913, Niels Bohr, un físico danés, dio una explicación de las observaciones de Rydberg. Escribió ecuaciones que describían el electrón de un átomo de hidrógeno moviéndose en torno al núcleo de una átomo, en orbitas circulares. Incluyó la suposición de que la energía electrónica está cuantizada. (LPF10, p. 176)

\section{Resultados}

En la Tabla 1 se presentan los resultados obtenidos para textos sugeridos en la formación inicial de profesores de química. En la categoría "Evidencias de la creatividad para la formulación de modelos científicos dentro de un marco teórico y como construcción humana", las referencias se hacen dentro de un contexto descriptivo y con tendencia hacia la retórica de conclusiones (NÍAZ, 2005) con un $28 \%$; una referencia incompleta con $27 \%$; no hay referencias en $17 \%$ lo que muestra una imagen acabada y una simplificación de la propuesta de Bohr; el 28 \% del modelo es enunciado dentro de marco histórico - epistemológico satisfactorio. En cuanto a la categoría epistemológica "Capacidad explicativa de los modelos", no se establece cuál es la importancia del modelo de Bohr y las causas que hicieron que la comunidad de especialistas se interesaran en corroborar el modelo mediante nuevas formulaciones e hipótesis, el $30 \%$ de las referencias es descriptiva e incompleta y el $70 \%$ no es referenciada; En lo relacionado con la categoría "Las evidencias del progreso científico en un contexto histórico - epistemológico acorde con un enfoque actual", la investigación muestra que este no es un aspecto al que se le dé importancia en la mayoría de los textos analizados, sólo el 12 $\%$ corresponde a un referencia satisfactoria; el $16 \%$ es referenciado en un marco de conclu- 
Moreno Ramírez, J. E.; Gallego Badillo, R.; Pérez Miranda, R.

sión; el 18\% muestra referencias incompletas y $54 \%$ de los aspectos analizados no son referenciados mostrando un enfoque positivista, en donde no se reconocen la actividad científica dentro una comunidad de especialistas, por otro lado los libros analizados presentan una imagen de ciencia acabada y lineal. No se evidencian las controversias ni las dificultades propias de todo proceso científico ocasionado deformaciones del accionar de las comunidades científicas.

Tabla 1. Análisis porcentual de los descriptores analizados en libros utilizados en formación inicial de profesores, LFP.

\begin{tabular}{|c|c|c|c|c|c|c|c|}
\hline Categoria & Subcategorias & $\mathbf{N}^{\circ}$ & RS $\%$ & $\mathbf{R} \%$ & $\mathbf{R} \mathbf{2} \%$ & SR\% & $\mathbf{r}$ \\
\hline Evidencias de la creatividad & Formulación del modelo & 1 & 00 & 43 & 57 & 00 & 00 \\
\hline para la formulación de modelos & analógico - simbólico & 2 & 00 & 85.7 & 14.3 & 00 & 00 \\
\hline científicos dentro de un marco & & 3 & 14.3 & 42.8 & 42.8 & 00 & 00 \\
\hline teórico y como construcción & Inserción de teorías para explicar & 4 & 14.3 & 14.3 & 14.3 & 57.14 & 00 \\
\hline \multirow[t]{2}{*}{ humana provisional } & y sustentar los hechos & 5 & 00 & 28.6 & 00 & 71.4 & 00 \\
\hline & & 6 & 00 & 00 & 00 & 00 & 100 \\
\hline \multirow[t]{2}{*}{$\begin{array}{l}\text { Capacidad explicativa de los } \\
\text { modelos científicos }\end{array}$} & $\begin{array}{l}\text { La utilidad y congruencia de los } \\
\text { modelos científicos }\end{array}$ & 7 & 00 & 14.3 & 57.1 & 28.6 & 00 \\
\hline & & 8 & 14.3 & 00 & 00 & 85.7 & 00 \\
\hline $\begin{array}{l}\text { Evidencias del progreso } \\
\text { científico en contexto histórico }\end{array}$ & $\begin{array}{l}\text { Antecedentes al modelo } \\
\text { semicuántico de Bohr }\end{array}$ & 9 & 00 & 14.3 & 85.7 & 00 & 00 \\
\hline - epistemológico acorde con & Aceptación, estudio, Modificación & 10 & 00 & 00 & 42.8 & 57.2 & 00 \\
\hline \multirow[t]{8}{*}{ un enfoque actual } & y sustitución de modelos & 11 & 00 & 42.8 & 00 & 00 & 57.2 \\
\hline & & 12 & 14.3 & 42.8 & 28.5 & 14.3 & 00 \\
\hline & $\begin{array}{l}\text { Controversias que se presentan } \\
\text { en las comunidades de }\end{array}$ & 13 & 00 & 00 & 00 & 100 & 00 \\
\hline & $\begin{array}{l}\text { especialistas } \\
\text { Modelos en competencia }\end{array}$ & 14 & 00 & 00 & 00 & 100 & 00 \\
\hline & & 15 & 00 & 00 & 00 & 100 & 00 \\
\hline & & 16 & 00 & 00 & 71.4 & 28.6 & 00 \\
\hline & Desarrollo del modelo dentro de & 17 & 00 & 28.6 & 57.1 & 14.3 & 00 \\
\hline & una comunidad de especialistas & 18 & 00 & 00 & 14.3 & 85.7 & 00 \\
\hline
\end{tabular}

La Tabla 2 muestra los resultados obtenidos en libros utilizados en educación media. Para la categoría "Evidencias de la creatividad para la formulación de modelos científicos dentro de un marco teórico y como construcción humana" El $5 \%$ de los textos analizados muestra una referencia satisfactoria de la elaboración del modelo; el $36 \%$ muestran referencias en el marco de la retórica de conclusiones, lo que permite inferirlos como un modelo definitivo; el $21 \%$ hace una referencia incompleta del modelo y el $38 \%$ no hace referencia a algún aspecto en particular en cuanto a su elaboración. En la segunda categoría "Capacidad explicativa de los modelos" los porcentajes encontrados son los siguientes; el $7 \%$ hacen una referencia satisfactoria; la referencia descriptiva es de $7 \%$; la referencia incompleta es de $29 \%$ y los libros que no dan ninguna referencia frente a la capacidad predictiva del modelo semi- 
El modelo semicuántico de Bohr ...

Tabla 2. Análisis porcentual de los descriptores analizados en libros utilizados en educación media. LEM.

\begin{tabular}{llccccc}
\hline \multicolumn{1}{c}{ Categoria } & \multicolumn{1}{c}{ Subcategorias } & $\mathbf{N}^{\mathbf{0}}$ & RS\% & $\mathbf{R} \%$ & RI\% & SR\% \\
\hline Evidencias de la creatividad para & Formulación del modelo analógico- & 1 & 20 & 20 & 50 & 10 \\
laformulación de modelos & simbólico & 2 & 40 & 20 & 40 & 00 \\
científicos dentrode un marco & & 3 & 30 & 60 & 10 & 00 \\
teórico y como & & 4 & 30 & 40 & 30 & 00 \\
construcciónhumana provisional & & 5 & 40 & 30 & 00 & 30 \\
& & 6 & 10 & 00 & 30 & 60 \\
& Inserción de teorías dentro de otra & 7 & 00 & 50 & 50 & 00 \\
Capacidad explicativa de los & para explicar y sustentar los hechos & & & & & \\
modelos científicos & La utilidad y congruencia de los & 8 & 10 & 10 & 10 & 70 \\
& modelos científicos & & & & & \\
Evidencias del progreso científico & Antecedentes al modelo semicuántico & 9 & 20 & 50 & 20 & 10 \\
en un contexto histórico - & de Bohr & & & & & \\
epistemológico acorde con un & Aceptación, estudio, modificación y & 10 & 00 & 00 & 40 & 60 \\
enfoque actual & sustitución de modelos & 11 & 30 & 10 & 10 & 50 \\
& & 12 & 60 & 30 & 10 & 00 \\
& Controversias que se presentan en las & 13 & 00 & 00 & 30 & 70 \\
& comunidades de especialistas & 14 & 00 & 00 & 00 & 100 \\
& Modelos en competencia & 15 & 00 & 00 & 00 & 100 \\
& Desarrollo del modelo dentro de una & 16 & 10 & 00 & 40 & 50 \\
& comunidad de especialistas & 17 & 00 & 70 & 30 & 00 \\
& & 18 & 00 & 20 & 00 & 80 \\
\hline
\end{tabular}

cuántico de N. Bohr es de $57 \%$; en cuanto a "El contexto histórico - epistemológico en el que se desarrolló el modelo", las referencias satisfactorias sólo alcanzan un porcentaje de $1 \%$; las referencias declarativas corresponden al $13 \%$; las referencias incompletas toman un $29 \%$; el porcentaje sin referencia relacionado afecta un $50 \%$ y las referencias erróneas corresponden a un $6 \%$, mostrando una versión de ciencia inadecuada para la formación de los estudiantes de educación media.

Con el fin de mostrar una tendencia en cuanto a la confiabilidad de los libros de texto en relación con la transposición del modelos semicuántico de $\mathrm{N}$. Bohr se asignan valores ponderados de 0,0 a 5.0 a cada criterio, se promediaron para obtener una tendencia de confiabilidad por cuanto algunos criterios son descritos, otros lo son parcialmente y otros no lo son, lo que hace que en su conjunto la propuesta presentada no corresponda a una referencia deseable a la elaborada en el interior de la comunidad científica.

El promedio general obtenido para los textos empleados en formación de profesores es bajo y no es aceptable por cuanto sus directos usuarios son los profesionales que se encargarán de la formación científica de los jóvenes que asisten a educarse en los colegios de educación secundaria (Tabla 3). En cuanto a los libros de texto utilizados en educación secundaria presentan valores mas bajos que los LFP, lo que implica que los estudiantes y docentes que utilizan este tipo de textos no reciben una versión de ciencia adaptada a las propuestas epistemológicas actuales ya citadas (Tabla 4). 
Moreno Ramírez, J. E.; Gallego Badillo, R.; Pérez Miranda, R.

Tabla 3. Tabla de resultados. RS: referencia satisfactoria; R referencia declarativa; RI: referencia incompleta; SR: sin referencia. Prom.: promedio. Se asignó un valor de $\mathbf{0 0}$ para SR; $\mathbf{3}$ para RI; $\mathbf{4}$ para $\mathbf{R}$ y $\mathbf{5 . 0}$ Para RS.

\begin{tabular}{llllllllllllllllllllll}
\hline & C1 & C2 & C3 & C4 & C5 & C6 & C7 & C8 & C9 & C10 & C11 & C12 & C13 & C14 & C15 & C16 & C17 & C18 & PROM \\
\hline LFP1 & RI & RI & R & R & R & SR & R & RI & R & RI & R & RS & SR & SR & SR & RI & R & R & $\mathbf{2 , 8 9}$ \\
LFP2 & RI & RS & R & R & SR & SR & RI & SR & R & RI & R & RS & SR & SR & SR & SR & RI & SR & $\mathbf{2 , 1 1}$ \\
LFP3 & RS & R & R & R & R & SR & R & R & RS & RI & RS & R & SR & SR & SR & RS & R & SR & $\mathbf{3 , 0 6}$ \\
LFP4 & RI & RI & R & R & SR & SR & R & SR & R & SR & SR & R & SR & SR & SR & SR & R & SR & $\mathbf{1 , 6 7}$ \\
LFP5 & R & RS & RI & SR & RS & RI & R & R & SR & SR & SR & RS & RI & SR & SR & RI & RI & SR & $\mathbf{2 , 3 3}$ \\
LFP6 & RI & RI & RS & RS & RS & SR & RI & SR & R & SR & SR & RI & RI & SR & SR & RI & RI & SR & $\mathbf{2 , 2 2}$ \\
LFP7 & RI & RS & RS & SR & R & RI & R & SR & RI & RI & SR & RS & RI & SR & SR & SR & R & SR & $\mathbf{2 , 3 3}$ \\
LFP8 & R & R & R & RS & RS & RS & RI & SR & RS & SR & RS & R & SR & SR & SR & SR & R & SR & $\mathbf{2 , 6 7}$ \\
LFP9 & SR & RI & R & RS & RS & SR & RI & SR & RI & SR & RS & RS & SR & SR & SR & SR & R & SR & $\mathbf{2 , 0 6}$ \\
LFP10 & RS & RS & RS & SR & SR & RI & RI & RS & R & SR & SR & RS & SR & SR & SR & RI & R & RS & $\mathbf{2 , 3 3}$ \\
\hline
\end{tabular}

Tabla 4. Tabla de resultados LFP. RS: referencia satisfactoria; R: referencia declarativa; RI: referencia incompleta; SR: sin referencia. r: error de referencia. Prom.: promedio. Se asignó un valor de $\mathbf{0 0}$ para SR y r; $\mathbf{3}$ para RI; $\mathbf{4}$ para R y 5.0 Para RS.

\begin{tabular}{cccccccccccccccccccc}
\hline & C1 & C2 & C3 & C4 & C5 & C6 & C7 & C8 & C9 & C10 & C11 & C12 & C13 & C14 & C15 & C16 & C17 & C18 & PROM \\
\hline LEM1 & RI & R & R & SR & SR & SR & R & SR & RI & SR & r & RI & SR & SR & SR & SR & RI & SR & $\mathbf{1 , 3 3}$ \\
LEM2 & R & R & R & RS & SR & SR & R & SR & RI & RI & R & R & SR & SR & SR & SR & RI & SR & $\mathbf{2 , 1 1}$ \\
LEM3 & R & R & RS & R & R & SR & RI & SR & RI & SR & r & R & SR & SR & SR & RI & RI & SR & $\mathbf{2 , 0 6}$ \\
LEM4 & RI & R & R & RI & R & SR & SR & SR & RI & RI & R & RS & SR & SR & SR & RI & R & SR & $\mathbf{2 , 2 2}$ \\
LEM5 & RI & R & RI & SR & SR & SR & RI & SR & R & SR & r & SR & SR & SR & SR & RI & SR & SR & $\mathbf{1 , 1 1}$ \\
LEM6 & RI & RI & RI & SR & SR & SR & RI & SR & RI & SR & R & RI & SR & SR & SR & RI & R & RI & $\mathbf{1 , 7 8}$ \\
LEM7 & R & R & RI & SR & SR & SR & SR & RS & RI & RI & r & R & SR & SR & SR & RI & RI & SR & $\mathbf{1 , 7 8}$
\end{tabular}

\section{Conclusiones}

El propósito de la investigación es la de contribuir en la reflexión que se está haciendo frente a los libros de texto que habitualmente se utilizan en la enseñanza de la ciencia química, en este sentido se aportan las siguientes conclusiones como elementos de discusión.

1 El análisis de los resultados permite evidenciar la preferencia que tienen los autores de los textos por mostrar los modelos ya elaborados sin abordar los problemas que los originaron y los que solucionaron; las dificultades y controversias a las que se enfrentaron las comunidades de especialistas cuando elaboraron el modelo. La trasposición didáctica que se hace del modelo semicuántico de Bohr en los textos utilizados en la formación de profesores presenta referencias que en conjunto, no son suficientemente satisfactorias para superar las visiones deformadas de la actividad científica, a saber: a) una imagen aproblemática y ahistórica, no se evidencian de forma clara los problemas que generaron la elaboración del modelo de Bohr y su progreso; las dificultades que enfrentó, las limitaciones que generaron controversias con otros científicos y su proceso regresivo 
El modelo semicuántico de Bohr ...

que llevaron a su abandono; b) una imagen rígida de la ciencia pues no se relacionan aspectos como la creatividad, la invención y la duda propia de todo proceso investigativo, los científicos que aportaron al modelo se nombran sin relación entre ellos se muestra una visión acumulativa y lineal que llevó al modelo de Bohr versión propia del empiro - positivismo, no se muestran los intentos para hacer del modelo un cuerpo coherente y estructurado desde los conceptos y la experiencia; sí se hace énfasis en aspectos de tipo experimental como la descomposición de la luz y los espectros de líneas y se emplean expresiones como descubrimiento de..., enigmas de... lo que supone un actividad en el marco de la observación y la experimentación, c) se promueve una visión positivista puesto que no se establece cuál fue la consistencia del modelo que hicieron que miembros de la comunidad de especialistas se interesara en su corroboración, se ignora el papel de nuevas formulaciones e hipótesis que fueron orientando la investigación científica para consolidar la propuesta; d) el modelo es simplificado y se dejan de lado algunos aspectos elaborados por Bohr y sus colaboradores mostrando sólo aquellas ideas que a juicio de los autores son importantes, no se muestran aspectos que en la mayoría de los casos permitieron que las ideas de Bohr lograran importantes victorias frente a las criticas de los contradictores

Si bien es cierto que los libros utilizados en la formación de profesores muestran un mejor acercamiento a la construcción del modelo, no es menos cierto que se alejan de una forma importante en lo que sería deseable en una transposición, la introducción de la historia de la ciencia en la formación de profesores es un factor fundamental en la superación de los obstáculos epistemológicos que subyacen a la enseñanza de las ciencias en la educación media, por ello con Garritz (2006) se afirma que los libros del siglo XXI deben superar al texto tradicional. La mera descripción de acontecimientos no es suficiente para formar profesores, éstos deben preguntarse acerca del origen del conocimiento, su construcción y progreso. Por otro lado si se acepta que hay unas correlaciones en la transposición didáctica entre saber científico - libros de texto - profesor - estudiante, se debe cuestionar entonces la calidad de la información que llega al estudiante de secundaria y con que versión se le acerca a la ciencia escolar.

2 En conexión con el párrafo anterior, las evidencias del progreso científico en un contexto histórico-epistemológico acorde con un enfoque actual esta ausente en la discusión en los libros para educación secundaria. La transposición didáctica que se hace del modelo semicuántico en estos textos promueve una versión de ciencia que no está acorde con las concepciones de la actividad científica aceptada actualmente, es de esperarse que los estudiantes que utilizan estos libros de texto y aún los docentes, tengan una concepción deformada de la forma como trabajan las comunidades científicas. En el caso de los profesores, esta imagen podría conllevar implicaciones didácticas que derivarían en un enfoque que puede promover una concepción en donde el empirismo, la observación ateórica, la descripción analítica y el aprendizaje memorístico sean el quehacer común en el aula de química, lo que reforzaría la actitud negativa hacia el estudio de las ciencias en general y de la química en particular por parte de los estudiantes en un momento histórico en el que se requieren personas científicamente alfabetizadas y con capacidad de decisión frente a problemas relacionados con la ciencia, la tecnología, el ambiente y la sociedad.

Es necesario que las comunidades de profesores se acerquen reflexiva y críticamente a los libros de texto con el fin de reformular su práctica didáctica en cuanto a la enseñanza de la ciencia química desde un punto de vista en el que primen las concepciones de ciencia acorde con modelos epistemológicos actuales, en donde se desmitifique al científico y la actividad 
Moreno Ramírez, J. E.; Gallego Badillo, R.; Pérez Miranda, R.

científica, sembrando en unos estudiantes el gusto y la posibilidad de estudiar y hacer ciencia y en otros la de alfabetizarlos científicamente teniendo en cuenta que como lo proponen Gallego Badillo, Pérez Miranda y Torres de Gallego (2004), la transposición requiere de tres elementos fundamentales: a) La interpretación que se puede hacer de los textos originales en donde se pusieron en consideración de la comunidad científica los modelos científicos; b) Las concepciones epistemológicas, didácticas y pedagógicas de quien hace la transposición didáctica y c) La intención curricular del grado y nivel del sistema educativo.

\section{Referencias}

BALMER, J. Note on the spectral lines of hydrogen. Annalen der Physik und Chemie, Leipzig, v. 261, n. 5, p. 80-85, 1885.

BOHR, N. The structure of the atom and the physical and chemical properties of the elements. Fysisk Tidsskrif, Oslo, v. 19, n. 4, p. 153, 1921.

. On the constitution of atoms and molecules. Philosophical Magazine, London, v. 26, Series 6, p. 1-15, 1913.

BRAKETT, F. Visible and infra-red radiation of hydrogen. Astrophysical Journal, London, v. 56, n. 3, p. 154-16, 1922.

CALDIN, E. F. The structure of chemistry in relation to the philosophy of science. Hyle: International Journal for Philosophy of Chemistry, Karlsruhe, v. 8, n. 2, p. 103-121, 2002. Disponível em: < http://www.hyle.org/journal/issues/8-2/caldin.html>. Acesso em: 10 set. 2009.

CAMACHO GONZÁLEZ, J. P.; PÉREZ MIRANDA, R. Análisis de la transposición didáctica de los conceptos calor y temperatura en los libros de texto para la enseñanza de la química. Tecné, Episteme y Didaxis, Bogotá, n. 17, p 117-128, $2005 .$.

.; GALLEGO BADILLO, R.; PÉREZ MIRANDA, R. La ley periódica: un análisis histórico epistemológico y didáctico de algunos textos de enseñanza. Educación Química, México, v. 18, n. 4, p. 278-288, 2007.

CHANG, R. Química. Bogotá: Mc Graw Hill, 2007.

CHEVALLARD, Y. La transposición didáctica: del saber sabio al saber enseñado. Buenos Aires: Aique, 1991.

CUÉLLAR FERNÁNDEZ, L.; GALLEGO BADILLO, R.; PÉREZ MIRANDA, R. El modelo atómico de E. Rutherford: del saber científico al conocimiento escolar. Enseñanza de las Ciencias, Barcelona, v. 26, n. 1, p. 43-52, 2008. 
El modelo semicuántico de Bohr ...

CUÉLLAR FERNÁNDEZ, L.; PÉREZ MIRANDA, R.; QUINTANILLA, M. La propuesta de Ernest Rutherford en los libros de texto en Colombia: un análisis desde la historia de las ciencias y la visión de transposición didáctica en ellos. Enseñanza de las Ciencias, Barcelona, v 22, n. extra, p. 1-6, 2005. Disponível em: <http://www.uc.cl/ sw_educ/educacion/grecia/plano/html/pdfs/linea_investigacion/ Analisis_de_Contenido_IAC/IAC_003.pdf>. Acesso em: 10 set. 2009.

DE BROGLIE, L. La física nueva de los cuantos. Buenos Aires: Losada, 1952.

DEL RE, G. Models and analogies in science. Hyle: Internacional Journal for Philosophy of Chemistry, Nápoles, v. 6, n. 1, p. 5-15, 2000. Disponível em: <http:// www.hyle.org/journal/issues/6/delre.htm>. Acesso em: 10 set. 2009.

FERNÁNDEZ, I.; GIL, D.; CARRASCOSA, J.; CACHAPUZ, A.; PRAIA, J. Visiones deformadas de la ciencia transmitidas por la enseñanza. Enseñanza de las Ciencias, Barcelona, v. 20, n. 3, p. 477-488, 2002.

GALLEGO BADILLO, R.; PÉREZ MIRANDA, R. Aprendibilidad, enseñabilidad y educabilidad en las ciencias experimentales. Revista educación y pedagogía, Medellín, v. 11, n. 25 , p. 89-117, 1998.

.; ___ _ GALLEGO TORRES, A.; TORRES DE GALLEGO, L. N. El objeto de saber de los químicos, formulación, modificación y abandono del modelo icónico inicial. Investigações em Ensino de Ciências, Porto Alegre, v. 11, n. 3, p. 365-381, 2006.

.;___ _ TORRES DE GALLEGO, L. N. La construcción escolar de las ciencias. Bogotá: Magisterio, 2004. (Colección Actualización Pedagógica).

.; ___ _ _ URIBE BELTRÁN, M.; CUÉLLAR FERNANDEZ, L.; AMADOR

RODRÍGUEZ, R. El concepto de valencia: su construcción histórica y epistemológica y la importancia de su inclusión en la enseñanza. Ciência \& Educação, Bauru, v. 10, n. 3, p. 571-583, 2004.

GÁNDARA GOMEZ, M.; GIL QUÍLEZ, M. J.; SANMARTÍ PUIG, N. Del modelo científico de "adaptación biológica" al modelo de "adaptación biológica" en los libros de texto de enseñanza secundaria obligatoria. Enseñanza de las Ciencias, Barcelona, v. 20, n. 2, p. 303-314, 2002.

GARRITZ, A. Naturaleza de la ciencia e indagación: cuestiones fundamentales para la educación científica del ciudadano. Revista Iberoamericana de Educación, Madrid, v. 42, n. 5, p. 127-152, 2006.

GEIGER, H.; MARSDEN, E. The laws of deflexion of a particles through large angles. Philosophical Magazine, London, v. 25, Series 6, p. 148, 1913.

On a diffuse reflection of the á-particles. Proceedings of the Royal Society of London, London, Series A, v. 82, n. 557, p. 495-500, 1909.

GONZÁLEZ RODRÍGUEZ, C.; GARCÍA BARROS, S.; MARTÍNEZ LOSADA, C. ¿A qué contenidos relacionados con la fotosíntesis dan más importancia los textos escolares de secundaria? Enseñanza de las Ciencias, Barcelona, v. 21, n. extra, p. 77-88, 2003. 
Moreno Ramírez, J. E.; Gallego Badillo, R.; Pérez Miranda, R.

GOUDSMIT, S.; UHLENBECK, G. E. Spinning electrons and the structure of spectra. Nature, London, v. 117, n. 2938, p. 264-265, 1926.

HEILBRON, J. L.; KUHN, T. S. The genesis of the Bohr atom. Historical Studies in the Physical Sciences, Berkeley, v. 1, n. 3-4, p. 211-290, 1969.

HEISENBERG, W. Encuentros y conversaciones con Einstein y otros ensayos. Madrid: Alianza, 1979.

The physical content of quantum kinematics and mechanics. Zeitschrift fur Physik, Leipzig, v. 43, n. 3-4, p. 172-178, 1927.

HERREÑO CHÁVEZ, J.; GALLEGO BADILLO, R.; PÉREZ MIRANDA, R. Transposición didáctica del modelo científico de Lewis-Langmuir. Revista Eureka sobre Enseñanza y Divulgación de la Ciencia, España, v. 7, n. 2, p. 527-543, 2010. Disponível em: <http://apac-eureka.org/revista/Volumen7/Numero_7_2/

Herre\%F1o_et_al_2010.pdf $>$. Acesso em: 8 out. 2010.

KUHN, T. S. La estructura de las revoluciones científicas. México: Fondo de Cultura Económica, 1972.

LAKATOS, I. La metodología de los programas de investigación científica. Madrid: Alianza, 1998.

LORENTZ, H. A. The motion of electrons in metallic bodies, III. In: ROYAL NETHERLANDS ACADEMY OF ARTS AND SCIENCES, 1905, Amsterdam.

Proceedings... Amsterdam, 1905. v. 7, p. 684-691. Disponível em: <http://

www.historyofscience.nl $/$ search $/$ detail.cfm?pubid $=673 \& v i e w=$ image $\&$ startrow $=1>$. Acesso em: 10 set. 2009.

LYMAN, T. An extension of the spectrum in the extreme ultra-violet, Nature, London, v. 93, n. 2323, p. 241, 1914.

NÍAZ, M. ¿Por qué los textos de química general no cambian y siguen una "retórica de conclusiones”? Educación Química, México, v. 16, n. 3, p. 410-415, 2005.

PÁEZ, Y.; RODRÍGUEZ, M. A.; NIAZ, M. Los modelos atómicos desde la perspectiva de la historia y filosofía de la ciencia: un análisis de la imagen reflejada por los textos de química de bachillerato. Investigación y Postgrado, Caracas, v. 19, n. 1, p. 51-77, 2004. Disponível em: <http://www.scielo.org.ve/scielo.php?pid=S1316-

00872004000100004\&script=sci_arttext $>$. Acesso em: 10 set. 2009.

PASCHEN, F. “'Zur Kenntnis ultraroter linienspektra. I. (bis Normalwellenlängen A.-E. 27000). Annalen der Physik, Berlin, v. 13, n. 13, p. 537-570, 1908.

PAULI, W. On the connexion between the completion of electron groups in an atom with the complex structure of spectra. Zeitschrift fur Physik, Berlin, v. 31, n. 1, p. 765-783, 1925.

PFUND, A. The emission of nitrogen and hydrogen in infrared. Journal of the Optical Society of America, Washington, v. 9, n. 3, p. 193, 1924. 
El modelo semicuántico de Bohr ...

RUTHERFORD, E. The scattering of alpha and beta particles by matter and the structure of the atom. Philosophical Magazine, London, v. 11, Serie 6, p. 669-688, 1911.

SCERRI, E. Philosophical confusion in chemical education research. Journal of Chemical Education, New York, v. 8, n. 5, p. 468-474, 2003.

SCHRÖDINGER, E. La nueva mecánica ondulatoria. Madrid: Biblioteca Nueva, 2001.

SOLBES, J.; TRAVER, M. J. La utilización de la historia de las ciencias en la enseñanza de la física y la química. Enseñanza de las Ciencias, Barcelona, v. 14, n. 1, p. 103-112, 1996.

THOMSON, J. J. On the structure of the atom. Philosophical Magazine, London, Series 6, v. 7, n. 39, p. 237-265. 1904.

. Catodhe rays. Philosophical Magazine, London, v. 44, Serie 5, p. 293-316, 1897.

TIPPENS, P. Física: conceptos y aplicaciones. Bogotá: Mc Graw Hill, 1988.

TOMASI, J. Towards "chemical congruence" of the models in theoretical chemistry. Hyle: An International Journal for Philosophy of Chemistry, Pisa, v. 5, n. 2, p. 79-115, 1999. Disponível em: < http://www.hyle.org/journal/issues/5/tomasi.htm>. Acesso em: 10 set. 2009.

ZEEMAN, P. The effect of magnetisation on the nature of light emitted by a substance. Nature, London, v. 55, n. 11, p. 347, 1897.

Artigo recebido em agosto de 2010 e aceito em novembro de 2010. 
Moreno Ramírez, J. E.; Gallego Badillo, R.; Pérez Miranda, R.

\section{Anexo A}

Tabla 5. Categorías, subcategorías y descriptores de análisis empleados en la investigación.

\begin{tabular}{|c|c|c|c|}
\hline Categoria & Subcategorias & $\mathrm{N}^{\circ}$ & Descriptor \\
\hline $\begin{array}{l}\text { Evidencias de la } \\
\text { creatividad para la } \\
\text { formulación de modelos } \\
\text { científicos como } \\
\text { construcción humana }\end{array}$ & $\begin{array}{l}\text { Formulación del modelo } \\
\text { analógico - simbólico } \\
\text { Inserción de una teoría } \\
\text { dentro de otra para } \\
\text { explicar los hechos }\end{array}$ & 5 & $\begin{array}{l}\text { El sistema atómico propuesto corresponde a un } \\
\text { átomo dehidrogeno con núcleo positivo y un } \\
\text { electrón de masa pequeña girando en una orbita } \\
\text { circular cerrada a una velocidad menor de la luz } \\
\text { El electrón puede pasar de un estado estacionario } \\
\text { a otrocon la emisión o absorción de energía } \\
\text { homogénea dando lugar a los espectro de líneas } \\
\text { respectivo } \\
\text { En condiciones ordinarias el electrón del } \\
\text { hidrogenopermanece en un estado fundamental } \\
\text { estacionario sin emisión de energía, siendo su } \\
\text { menor valor igual a } 1 \\
\text { La radiación emitida durante la transición de un } \\
\text { estado aotro es homogénea la cual está dada por } \\
W t_{2}-\text { W } t_{1}=\text { hv } \\
\text { El momento angular de un electrón que gira en una } \\
\text { orbita circular alrededor de un núcleo positivo en } \\
\text { un átomo normal es igual nh/2 } \pi \text {, } \\
\text { En un átomo en equilibrio dinámico los estados } \\
\text { estacionarios de los electrones se pueden explicar } \\
\text { desde la mecánica clásica; } \\
\text { Las transiciones de un estado estacionario a otro } \\
\text { se explica por la teoría de Planck }\end{array}$ \\
\hline $\begin{array}{l}\text { Capacidad explicativa de } \\
\text { los modelos científicos }\end{array}$ & $\begin{array}{l}\text { La utilidad y congruencia } \\
\text { de los modelos } \\
\text { científicos }\end{array}$ & 7 & $\begin{array}{l}\text { El modelo explica las líneas de emisión de Balmer } \\
\text { y Paschen y predecir otras (Brakett, Pfund) para el } \\
\text { hidrogeno } \\
\text { El modelo semicuántico propuesto por Bohr } \\
\text { permite deducir la ecuación empírica de Rydberg }\end{array}$ \\
\hline $\begin{array}{l}\text { Evidencias del progreso } \\
\text { científico en un contexto } \\
\text { histórico-epistemológico } \\
\text { actual }\end{array}$ & $\begin{array}{l}\text { Antecedentes al modelo } \\
\text { semicuántico de Bohr } \\
\text { Aceptación, estudio, } \\
\text { modificación y sustitución } \\
\text { de modelos } \\
\text { Controversias que se } \\
\text { presentan en las } \\
\text { comunidades de } \\
\text { especialistas } \\
\text { Modelos en competencia } \\
\text { Desarrollo del modelo } \\
\text { dentro de una comunidad } \\
\text { de especialistas }\end{array}$ & $\begin{array}{l}12 \\
13\end{array}$ & $\begin{array}{l}\text { Teorías de la electrodinámica clásica, Teoría de } \\
\text { Planck y efecto fotoeléctrico } \\
\text { Los trabajos de Bohr se realizaron con base en el } \\
\text { modelo atómico de Rutherford descartando el } \\
\text { modelo de J. J.Thomson } \\
\text { La inestabilidad del sistema de electrones del } \\
\text { átomo de Rutherford, lleva a Bohr hacia la } \\
\text { formulación del modelo semicuántico } \\
\text { Emerge el modelo mecánico ondulatorio } \\
\text { Las controversias de Bohr con Fowler, De Broglie y } \\
\text { Schrödinger } \\
\text { Las inconsistencias del modelo de Bohr promueve } \\
\text { la propuesta de De Broglie } \\
\text { El modelo semicuántico de Bohr, rivaliza con los } \\
\text { modelos de Thomson y Rutherford } \\
\text { Sommerfeld hizo aportes fundamentales al } \\
\text { incorporar la relatividad de Einstein en los cálculos } \\
\text { del modelo de Bohr. } \\
\text { Los aportes de Pauli: el principio de exclusión; el } \\
\text { trabajo de Gousdmit y Uhlenbeck: spin del electrón } \\
\text { El trabajo de Bohr se desarrolla al interior del } \\
\text { grupo de Manchester liderado por Rutherford }\end{array}$ \\
\hline
\end{tabular}


El modelo semicuántico de Bohr ...

\section{Anexo B}

Libros de Educación Superior (LFP)

LFP1 - Babor, J.; Aznárez, J. Química general moderna: una introducción a la Química física y a la Química descriptiva superior. Editorial Marín, 1983.

LFP2 - Brown, T.; LeMay, B. Química: la ciencia central. 7. ed. Prentice Hall, 2004.

LFP3 - Cruz-Garritz, D.; Chamizo, J.; Garrita, A. Estructura atómica: un enfoque químico. Fondo Educativo Interamericano, 1986.

LFP4 - Chang, R. Química. 6. ed. Mc Graw Hill, 1999 y 2007.

LFP5 - Cotton, A.; Wilkinson, G. Química inorgánica básica. Limusa, 2006.

LFP6 - Mahan, B.; Myers, R. Química: curso universitario. Addison-Wesley, 1977 y 1990.

LFP7 - Masterton, W. L.; Slowinski, E. J.; Stanitski, L. C. Química general superior. 6. ed. Mc Graw Hill, 1987.

LFP8 - Petrucci, R.; Harwood, W.; Herring, G. Química general. Prentice Hall, 2002.

LFP9 - Sienko, M.; Plane, R. Química: principios y aplicaciones. McGraw-Hill, 1986.

LFP10 - Whitten, K.; Davis, R.; Peck, L. Química general. 5. ed. Mc Graw Hill, 1998.

\section{Libros de Educación Media (LEM)}

LEM1 - Arena, H. Fundamentos de quimica. Thomson, 2005.

LEM2 - Cárdenas, F.; Gálvez, C. A. Química y Ambiente I. Mc Graw Hill, 2003.

LEM3 - Herrera, S.; Barreto, A.; Torres, I.; De Cavijo, E. Quimica 1. Norma, 1984.

LEM4 - Manco, L. F. Química 10: general e inorgánica. Migema, 1993.

LEM5 - Mondragón, C.; Peña, L.; Sánchez de Escobar, M.; Fernández, M. Química I.

Santillana, 2001.

LEM6 - Montoya Potes, R. Química moderna. Bedout, 1990.

LEM7 - Poveda, V. J. Química 10. 6. ed. Educar Editores, 2001. 